SHORT REPORT

\title{
The retroperitoneal surface in distal caecal and proximal ascending colon carcinoma: the Cinderella surgical margin?
}

\author{
A C Bateman, N J Carr, B F Warren
}

J Clin Pathol 2005;58:426-428. doi: 10.1136/icp.2004.019802

Background: Mesorectal margin tumour involvement is a predictor of local recurrence in rectal carcinoma and an indication for postoperative radiotherapy in suitable patients. However, the prevalence of non-peritonealised surgical margin involvement in ascending colon carcinoma is unknown.

Aims: To test the hypothesis that retroperitoneal surgical margin (RSM) tumour involvement occurs in distal caecal and proximal ascending colon carcinoma.

Methods/Results: One hundred right hemicolectomy specimens, removed for adenocarcinoma of the caecum or proximal ascending colon, were studied. During routine specimen dissection, at least one additional tissue block was taken to include the tumour and the RSM. The tumour distance from the RSM was recorded. RSM tumour involvement was present in seven cases (7\%). Direct (non-nodal) RSM tumour involvement (five cases) only occurred in posterior or circumferential tumours.

Conclusions: RSM tumour involvement occurs within a considerable number of distal caecal and proximal ascending colon carcinomas. The rate of RSM tumour involvement identified here is similar to a previously published local recurrence rate of $10 \%$ in caecal carcinoma, suggesting that RSM tumour involvement may be a predictor of recurrence in these tumours. Therefore, patients with distal caecal or proximal ascending colon carcinoma and RSM tumour involvement may benefit from postoperative radiotherapy.

$\mathrm{T}$ umour involvement of the mesorectal excision plane is a well established and powerful predictor of local recurrence in rectal carcinoma. ${ }^{1}$ Local recurrence of rectal carcinoma is associated with a poor prognosis. Total mesorectal excision aims to reduce the rate of mesorectal excision plane tumour involvement, and successful surgery of this type is associated with reduced rates of local recurrence. ${ }^{2}$ Surgical margin involvement is an adverse prognostic factor in colonic carcinoma. ${ }^{2 a}$ There is evidence that postoperative radiotherapy improves survival in rectal cancer and in advanced colonic cancer. ${ }^{3-5}$ A large multicentre study (the MRC-CRO7 trial) is currently assessing the value of postoperative radiotherapy for patients undergoing rectal excision for colorectal cancer in whom the tumour lies within $1 \mathrm{~mm}$ of the mesorectal excision plane.

"We hypothesised that tumours arising within the posterior wall of the distal caecum and proximal ascending colon may extend through the intestinal wall and involve this retroperitoneal surgical margin"
The distal caecum and proximal ascending colon may bear a short mesentery, but in some individuals no mesentery is present, and the posterior aspect of the ascending colon is therefore a non-peritonealised area of variable size. ${ }^{6}$ Therefore, we hypothesised that tumours arising within the posterior wall of the distal caecum and proximal ascending colon may extend through the intestinal wall and involve this retroperitoneal surgical margin (RSM). The incidence of RSM tumour involvement in tumours of the distal caecum and proximal ascending colon and its implications for patient outcome and postoperative management are currently unknown.

The aim of our study was to test the hypothesis that RSM tumour involvement occurs in carcinoma of the distal caecum and proximal ascending colon, using standard histopathological techniques.

\section{MATERIALS AND METHODS}

One hundred right hemicolectomy specimens removed from patients with adenocarcinoma of the distal caecum or proximal ascending colon were studied. The specimens were dissected and underwent histopathological reporting using standard histopathological techniques, as described within the Royal College of Pathologists' minimum dataset guidelines for colorectal carcinoma. ${ }^{7}$ In addition, the position of the tumour within the caecum was recorded.

In cases where a retrocaecal "bare area" (and therefore a RSM) was present, at least one additional tissue block was taken to include the closest approach of the tumour to the RSM. During subsequent reporting, the minimum distance between the advancing edge of the tumour and the RSM was measured using a microscope stage vernier scale (fig 1$).{ }^{8}$ RSM tumour involvement was defined as being present if the minimum distance between the tumour and the RSM was less than $1 \mathrm{~mm} .^{7}$ In this situation, "tumour" could represent either direct growth of the primary adenocarcinoma or the presence of metastatic tumour within a retrocolonic lymph node.

\section{RESULTS}

One hundred right hemicolectomy specimens, removed for adenocarcinoma of the distal caecum or proximal ascending colon, were studied. Table 1 gives the distribution of tumour position (for the 50 cases in which this information was available). The tumour involved the posterior wall of the distal caecum or proximal ascending colon in 34 of these 54 cases. RSM tumour involvement, defined as the presence of tumour within $1 \mathrm{~mm}$ of the RSM, was identified in seven cases. In five of these cases, RSM tumour involvement resulted from direct RSM encroachment by the primary tumour. In the remaining two cases, RSM tumour

Abbreviations: RSM, retroperitoneal surgical margin 


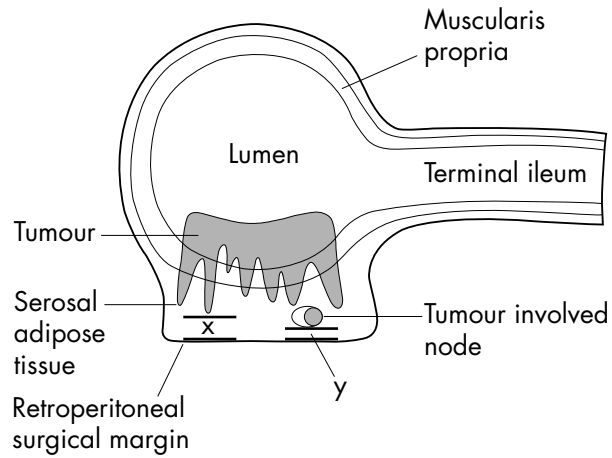

Figure 1 Line diagram of a horizontal section through a caecal adenocarcinoma showing the position at which the distance between the advancing edge of the tumour and the retroperitoneal surgical margin was measured.

involvement was recorded as a result of the presence of a tumour involved lymph node within $1 \mathrm{~mm}$ of the RSM. Within the 50 cases of known tumour position, RSM tumour involvement occurred only in circumferential tumours or those involving primarily the posterior wall of the distal caecum or proximal ascending colon.

\section{DISCUSSION}

In our study, we found RSM tumour involvement in 7\% of adenocarcinomas of the distal caecum and proximal ascending colon. This feature was identified using standard histopathological techniques. RSM tumour involvement occurred more often as a result of direct growth of the primary tumour than because of the presence of a tumour involved lymph node close to the RSM.

Mesorectal excision plane tumour involvement is a well known risk factor for local recurrence in rectal adenocarcinoma, imparting a poor prognosis. ${ }^{1}$ Total mesorectal excision is now a widely used surgical procedure, and results in a decrease in the local recurrence rate because the incidence of mesorectal excision plane involvement is reduced. ${ }^{2}$ Suitable patients in whom mesorectal excision plane tumour involvement is identified after histopathological examination of the resected tumour are now considered for postoperative radiotherapy.

Surgical margin tumour involvement is associated with impaired survival in Dukes' $\mathrm{B}$ colonic adenocarcinoma. ${ }^{2 a}$ Local recurrence occurs in $10 \%$ of adenocarcinomas treated by right hemicolectomy alone. ${ }^{9}{ }^{10}$ Although chemotherapy is usually considered for suitable patients with lymph node metastases, postoperative radiotherapy is not a commonly used adjuvant treatment for these tumours. The anatomy of the proximal colon is variable, with a retrocolonic nonperitonealised bare area present in many patients. We have shown that adenocarcinoma may involve this surgical margin if the tumour is posterior or circumferential in nature. Alternatively, tumour may closely approach the RSM if metastatic adenocarcinoma is present within a tumour involved retrocolonic lymph node, which itself lies close to or at this surgical margin. A distance of $1 \mathrm{~mm}$ between the tumour and RSM was chosen to indicate RSM tumour involvement in accordance with the Royal College of Pathologists' minimum dataset guidelines. ${ }^{7}$

\footnotetext{
"Retroperitoneal surgical margin (RSM) tumour involvement occurred more often as a result of direct growth of the primary tumour than because of the presence of a tumour involved lymph node close to the RSM"
}

Table 1 Distribution of tumour position within the caecum/proximal ascending colon

\begin{tabular}{ll}
\hline Tumour position & Number of cases \\
\hline Circumferential & 18 \\
Posterior wall & 15 \\
Medial or lateral wall & 7 \\
Anterior wall & 6 \\
Pole of caecum & 4 \\
\hline
\end{tabular}

It is currently unclear whether RSM tumour involvement is directly related to local tumour recurrence in distal caecal and proximal ascending colon adenocarcinomas. However, the similarity between the rate of RSM tumour involvement identified in our study and the previously reported rate of local tumour recurrence in right colonic adenocarcinoma suggests that there may be a link between this histopathological feature and disease recurrence.

Although a retrocolonic non-peritonealised bare area is not uncommonly identified, the amount of serosal adipose tissue present within this area is small. This means that complete local surgical excision of posterior or circumferential adenocarcinomas of the distal caecum or ascending colon may be difficult or impossible if there is significant tumour extension into this retrocolonic tissue.

Postoperative radiotherapy is of confirmed value in reducing local tumour recurrence rates in rectal adenocarcinoma. ${ }^{3}$ It is possible that radiotherapy to the posterior abdominal wall in the position of the caecal/ascending colon bed may produce a similar survival benefit in cases where RSM tumour involvement is identified within resected right hemicolectomy specimens. Two previous studies have suggested that postoperative radiotherapy may improve survival in advanced colonic adenocarcinoma, including tumours within the caecum and ascending colon. ${ }^{34}$ Both of these studies used a modification of the Astler-Coller staging system, which is not now recommended for use in the UK. ${ }^{11}$ Both studies found survival benefit with radiotherapy for patients in whom direct tumour involvement of adjacent tissues had occurred. Unlike our study, the precise nature of adjacent tissue tumour involvement was not stated; in particular, no distinction was made between tumour involvement of peritonealised and non-peritonealised surfaces. Much larger prospective multicentre studies are warranted to test the hypotheses that RSM tumour involvement increases the risk of local tumour recurrence in adenocarcinoma of the caecum and proximal ascending colon and that postoperative radiotherapy may improve outcome in cases where tumour closely approaches the RSM. Such studies

\section{Take home messages}

- We found that retroperitoneal surgical margin (RSM) tumour involvement occurs within a considerable number of distal caecal and proximal ascending colon carcinomas

- The 7\% rate of RSM tumour involvement found in our study is similar to a previously published local recurrence rate of $10 \%$ in caecal carcinoma, suggesting that RSM tumour involvement may be a predictor of recurrence in these tumours

- Patients with distal caecal or proximal ascending colon carcinoma and RSM tumour involvement might benefit from postoperative radiotherapy 
should operate under a carefully designed protocol, which would include a very clear description of the anatomy of the ascending colon and the distance of the primary tumour or tumour involved lymph nodes from the RSM in each patient. A follow up period of at least five years would also be necessary.

\section{Authors' affiliations}

A C Bateman, N J Carr, Department of Cellular Pathology, Southampton University Hospitals NHS Trust, Southampton S016 6YD UK

B F Warren, Department of Cellular Pathology, John Radcliffe Hospital, Oxford OX3 9DU, UK

Correspondence to: $\operatorname{Dr}$ A C Bateman, Department of Histopathology, Mailpoint 2, Level E, South Block, Southampton General Hospital, Tremona Road, Southampton SO16 6YD, UK; adrian.bateman@suht. swest.nhs.uk

Accepted for publication 8 September 2004

\section{REFERENCES}

1 Quirke P, Dixon MF, Durdey P, et al. Local recurrence of rectal adenocarcinoma due to inadequate surgical resection: histopathological study of lateral tumour spread and surgical excision. Lancet 1986;ii:996-9.
2 MacFarlane JK, Ryall RD, Heald RJ. Mesorectal excision for rectal cancer. Lancet 1993;341:457-60.

2a Petersen VC, Baxter KJ, Love SB, et al. Identification of objective pathological prognostic determinants and models of prognosis in Dukes' B colon cancer. Gut 2002;51:65-9.

3 Colorectal Cancer Collaborative Group. Adjuvant radiotherapy for rectal cancer: a systematic overview of 8507 patients from 22 randomised trials. Lancet 2001;358:1291-304.

4 Willett CG, Fung CF, Kaufman DS, et al. Postoperative radiation therapy for high-risk colon carcinoma. J Clin Oncol 1993;11:1112-17.

5 Amos EH, Mendenhall WM, McCarty PJ, et al. Postoperative radiotherapy for locally advanced colon cancer. Ann Surg Oncol 1996;3:431-6.

6 Burroughs SH, Williams GT. ACP Best Practice No 159. Examination of large intestine resection specimens. J Clin Pathol 2000;53:344-9.

7 Quirke P, Williams GT. Standards and minimum datasets for reporting common cancers. Minimum dataset for colorectal carcinoma histopathology reports. London: Royal College of Pathologists, 1998.

8 Warren BF, Davies JD. Pierre Vernier's invention: a neglected tool of our trade. Histopathology $1991 ; 18: 361-2$.

9 Tong D, Russell AH, Dawson LE, et al. Adenocarcinoma of the cecum: natural history and clinical patterns of recurrence following radical surgery. Int $J$ Radiat Oncol Biol Phys 1983;9:357-60.

10 Kopelson G. Adjuvant postoperative radiation therapy for colorectal carcinoma above the peritoneal reflection. II. Antimesenteric ascending and descending colon and rectum. Cancer 1983:52:633-6.

11 Gunderson LL, Sosin H, Levitt S. Extrapelvic colon-areas of failure in a reoperation series: implications for adjuvant therapy. Int J Radiat Oncol Biol Phys 1985;11:731-41. 